Ergod. Th. \& Dynam. Sys. (1984), 4, 541-557

Printed in Great Britain

\title{
Lower entropy factors of sofic systems
}

\author{
MIKE BOYLE \\ Department of Mathematics, University of Washington, Seattle, Washington 98195 , \\ USA
}

(Received 23 November 1982 and revised 15 September 1983)

\begin{abstract}
A mixing subshift of finite type $T$ is a factor of a sofic shift $S$ of greater entropy if and only if the period of any periodic point of $S$ is divisible by the period of some periodic point of $T$. Mixing sofic shifts $T$ satisfying this theorem are characterized, as are those mixing sofic shifts for which Krieger's Embedding Theorem holds. These and other results rest on a general method for extending shift-commuting continuous maps into mixing subshifts of finite type.
\end{abstract}

\section{Introduction}

Wolfgang Krieger ([5]) has proved a striking embedding theorem: if $S$ is a mixing subshift of finite type (MSFT), and $T$ is any subshift with $h(T)<h(S)$ which satisfies a trivially necessary periodic condition, then $T$ is isomorphic to a subshift of $S$. The trivial condition is that for all $j, \pi_{j}(T) \leq \pi_{j}(S)$, where $\pi_{j}(T)=\left|P_{j}(T)\right|$ and

$$
P_{j}(T)=\left\{x: T^{j} x=x, T^{i} x \neq x \text { for } 0<i<j\right\} .
$$

This result suggests an optimistic conjecture: if $S$ and $T$ are MSFT's with $h(T)<h(S)$ which satisfy some trivially necessary periodic condition, then $T$ is a factor of $S(S \downarrow T)$. The conjecture is true. (In fact, $S$ and $T$ need only be irreducible subshifts of finite type.) The trivial condition $(S \stackrel{\text { per }}{\longrightarrow} T)$ is that the period of any periodic point of $S$ is divisible by the period of some periodic point of $T$. Bruce Kitchens and Brian Marcus have proved this theorem independently, finding essentially the same proof which leads naturally to the other results of this paper.

We prove the theorem in two steps. First we cover $T$ with an MSFT of equal entropy which by Krieger's theorem is isomorphic to a subshift $\bar{S}$ of $S$. (Krieger has already done this in [5]; we give a more elaborate lemma for later use.) Then we extend the factor map $\bar{S} \downarrow T$ to a factor map $S \downarrow T$ by means of a general extension lemma. If $\bar{\phi}$ is a continuous shift-commuting map from a subshift $\bar{S}$ into an MSFT $T$, where $\bar{S}$ is a subshift of a subshift $S$ and $S \stackrel{\text { per }}{\longrightarrow} T$, then the lemma extends $\bar{\phi}$ to a continuous shift-commuting map from $S$ into $T$. The lemma's proof is a straightforward adaptation of the marker method Krieger used to prove his embedding theorem.

As one corollary, we find that irreducible subshifts of finite type with the same zeta function have the same (sofic) factors of lower entropy. As another, we find that a sofic shift $S$ factors onto an MSFT $T$ of lower entropy if $S \stackrel{\text { per }}{\longrightarrow} T$. These results fail in the larger class of sofic shifts. Examples lead us to isolate a class of periodic points we call receptive points. Where the existence of an embedding or factor map from $S$ to a mixing SFT $T$ is guaranteed by an entropy inequality and 
a sufficiency of periodic points in $T$, the existence of the map into a mixing sofic shift $T$ will be guaranteed by the entropy inequality and a sufficiency of receptive periodic points in $T$. These results let us characterize those mixing sofic shifts which behave like MSFT's with respect to higher-entropy sofic extensions (receptive shifts) and those which behave like MSFT's with respect to embeddings (inclusive shifts). A mixing sofic shift is receptive if and only if the period of any periodic point is divisible by the period of some receptive point. A mixing sofic shift is inclusive if and only if every periodic point is receptive.

\section{Definitions and background}

Let a finite set of $n$ elements, $\{1,2, \ldots, n\}$ say, be given the discrete topology. The (full) $n$-shift is the homeomorphism obtained by furnishing the set $\prod_{i \in \mathbf{Z}}\{1,2, \ldots, n\}_{i}$ with the product topology and the left shift transformation. A subshift $S$ is the restriction of some $\boldsymbol{n}$-shift to a closed shift-invariant subset. We shall use the same symbol to denote the underlying space of a subshift and the restriction of the left shift transformation to this space. We will also often refer to a subshift as a shift. The alphabet $A(S)$ of the subshift $S$ is the subset of $\{1,2, \ldots, n\}$ consisting of those elements that appear in some point of $S$. An element of $A(S)$ is a symbol of $S$. If $c_{1}, c_{2}, \ldots, c_{l} \in A(S)$ are such that the string $C=c_{1} c_{2} \ldots c_{l}$ appears in some point of $S$, then $C$ is a word or block of $S$, whose length $l$ may be denoted by $|C|$. We also use the terms $S$-word and $S$-block. We let $B_{l}(S)$ denote the set of $S$-words of length $l$ (the l-blocks of $S$ ), while $B(S)$ denotes the set of all $S$-words. If $A$ is an $l$-block, then $A^{n}$ is the $n l$-block formed by repeating $A n$ times. We use $A^{\infty}$ to denote an infinite string of symbols formed by repeating $A ; A^{\infty}$ may extend infinitely to either side or both, by context.

Any subshift is obtained from some $n$-shift by discarding from the domain all sequences in which any of a given collection of words occurs. In general, the collection may be countably infinite. If a subshift $S$ may be obtained by disallowing only a finite collection of words, then $S$ is a subshift of finite type (SFT). If every word in the forbidden collection has length less than or equal to $k+1$, then $S$ is $k$-step. Equivalently, $S$ is a $k$-step SFT if the points in $S$ are all the sequences $x$ such that, for every $i \in \mathbb{Z}, x_{i} \ldots x_{i+k} \in B_{k+1}(S)$. A 1 -step SFT $S$, say with alphabet $\{1,2, \ldots, k\}$, may be determined by a $k$ by $k$ matrix with entries in $\{0,1\}$ such that $A_{i j}=1$ if and only if $i j \in B_{2}(S)$. Similarly, $S$ is determined by the graph whose vertices are the elements of $A(S)$ : there is an arc from $i$ to $j$ in the graph if and only if $i j \in B_{2}(S)$. If $i j \in B_{2}(S)$, then $i$ is a predecessor of $j$, and $j$ is a follower of $i$.

If $S$ and $T$ are subshifts, and $\phi$ is a continuous map such that $\phi S=T \phi$, then $\phi$ is shift-commuting, $\phi$ is a code, and we write $\phi: S \rightarrow T$. If $\phi$ is surjective, then $T$ is a factor of $S, S$ is a cover (or lift or extension) of $T, \phi$ is a factor map and we write $\phi: S \downarrow T$, or just $S \downarrow T$. If $\phi$ is bijective, then $S$ and $T$ are isomorphic or conjugate, and $\phi$ is a conjugacy. If $\phi_{1}$ and $\phi_{2}$ are conjugacies, while $\psi_{1}$ and $\psi_{2}$ are shiftcommuting maps such that $\phi_{2} \psi_{1}=\psi_{2} \phi_{1}$, then $\psi_{1}$ and $\psi_{2}$ are isomorphic. Typically isomorphic maps are interchangeable for our purposes, and we announce an exchange with the incantation 'by recoding, we may assume... The $n$-block 
presentation $S^{[n]}$ of a subshift $S$ is the subshift whose alphabet is $B_{n}(S)$ and whose domain is

$\left\{\bar{x}\right.$ : There exists $x \in S$ such that for all $\left.i \in \mathbb{Z}, \bar{x}_{i}=x_{i} \ldots x_{i+n-1}\right\}$.

$S^{[n]}$ is conjugate to $S$ by the natural map. If $S$ is a $k$-step SFT and $n \geq k$, then $S^{[n]}$ is a 1-step SFT. A $k$-block map $\phi$ from $S$ to $T$ is defined by a map $\hat{\phi}$ from $B_{k}(S)$ to $A(T)$ by $(\phi x)_{n}=\hat{\phi}\left(x_{n} \ldots x_{n+k-1}\right)$. The Curtis-Hedlund-Lyndon Theorem asserts that any continuous shift-commuting $\phi$ is a block map composed with some power of the shift; so, by recoding we may assume that $\phi$ is a 1-block map.

An SFT $S$ is irreducible if for any $S$-words $A$ and $C$, there is an $S$-word $B$ such that $A B C$ is an $S$-word. If for given $A$ and $C$, there exists $N$ such that this $B$ may be chosen to have any length greater than or equal to $N$, then $S$ is topologically mixing. If this $N$ may be chosen uniformly for all $A$ and $C$, then $N+1$ is a transition length for $S$. Every mixing SFT (MSFT) has a transition length. If $S$ is an irreducible SFT, then there exists a positive integer $k$, the (ergodic) period of $S$, and $k$ sets $E_{1}, \ldots, E_{k}$ (the cyclically moving subsets of $S$ - see $[1, \S 3]$ ) with the following properties:

(1) $k$ is the greatest common divisor of the periods of the periodic points of $S$;

(2) the restriction of $S^{k}$ to each $E_{i}$ is an MSFT;

(3) $S$ is a disjoint union of the $E_{i}$;

(4) for each $E_{i}, S E_{i}=E_{i+1}$, where the subscripts are interpreted $\bmod k$.

If $E$ is one of the cyclically moving subsets, it is easy to see that a shift-commuting map $\left.S^{k}\right|_{E} \rightarrow T^{k}$ has a unique extension $\phi$ to all of $S$ such that $\phi: S \rightarrow T$.

The topological entropy of a subshift $S, h(S)$, is

If $S$ is an SFT, then also

$$
\lim _{n} \frac{1}{n} \log \left|B_{n}(S)\right|
$$

$$
h(S)=\lim _{n} \frac{1}{n} \log \left|P_{n}(S)\right| .
$$

If $S \downarrow T$, then $h(S) \geq h(T)$. Any SFT contains an irreducible SFT of equal entropy. For more on subshifts and SFT's, see [1] and [2]. (These references use the symbol $\sigma$ for the shift map on any domain; subshifts are distinguished by the symbol which names the domain.)

A subshift is sofic if it is a factor of an SFT([8]). If $T$ is sofic, then there is a 1-step SFT $S$ and a factor map $\phi: S \downarrow T$ with the following properties (see [3]):

(1) $\phi$ is 1 -block;

(2) $\phi$ is bounded-to-one: that is, there exists $N$ such that no point of $T$ has more than $N$ preimages;

(3) $\phi$ is $1-1$ a.e.: that is, any doubly transitive point of $T$ has a unique preimage;

(4) $T$ has a magic word: that is, there exists a word $W=w_{1} \ldots w_{k}$ and a number $I, 1 \leq I \leq k$, such that for any $x, \bar{x}$ in $S$, if

then $x_{i+1}=\bar{x}_{i+I}$;

$$
(\phi x)_{i+1} \ldots(\phi x)_{i+k}=W=(\phi \bar{x})_{i+1} \ldots(\phi \bar{x})_{i+k}
$$

(5) if $T$ is mixing, then $S$ is mixing. 
In the sequel, magic words are defined implicitly with respect to some fixed $\phi$ satisfying the properties above; the particular $\phi$ never matters. A magic word $W$ has a very useful intersection property, which we use repeatedly in $\S 3$ : if $B W$ and $W C$ are $T$-words, then $B W C$ is a $T$-word. (To see this, notice that if $\phi$ takes $b_{1} \ldots b_{i}^{\prime} w_{1}^{\prime} \ldots w_{k}^{\prime}$ to $B W$ and $w_{1}^{\prime \prime} \ldots w_{k}^{\prime \prime} c_{1}^{\prime \prime} \ldots c_{i}^{\prime \prime}$ to $W C$, then $w_{I}^{\prime}=w_{I}^{\prime \prime}$; since $S$ is 1-step, $b_{1}^{\prime} \ldots b_{i}^{\prime} w_{1}^{\prime} \ldots w_{I}^{\prime} w_{I+1}^{\prime \prime} \ldots w_{k}^{\prime \prime} c_{1}^{\prime \prime} \ldots c_{j}^{\prime \prime}$ is in $B(S)$.) Any word containing a magic word must be a magic word, so most long $T$-words must be magic words. From these observations, Marcus ([7]) proved a theorem which is fundamental to all our sofic results. We find it convenient to use a slight specialization of his theorem. Given a sofic shift $T$, fix a magic word $W$, and let $T_{k}$ be the $(k-1)$ step SFT whose $k$-blocks are those $T$-words $b_{1} \ldots b_{k}$ such that $W$ occurs in $b_{2} \ldots b_{k}$. Then $T_{k}$ is a subshift of $T$ and $\lim h\left(T_{k}\right)=h(T)$.,

If $T$ is mixing, then for large enough $k, T_{k}$ is also mixing. To see this, recall that $\phi: S \downarrow T$ is a 1 -block map and $S$ is mixing. Thus if $N$ is a transition length for $S$, then it is also a transition length for $T$ : so there exist $B$ in $B_{N-1}(T)$ and $C$ in $B_{N}(T)$ such that $W B W$ and $W C W$ are in $B(T)$. Now suppose $k \geq N+2|W|$. Then the periodic orbits defined by $W B$ and $W C$ (which exist by the intersection property of magic words) must be in $T_{k}$. That is, the greatest common divisor of the periods of the periodic points of $T_{k}$ is 1 ; if $T_{k}$ is irreducible, then it must be mixing. But any word of $T_{k}$ occurs in some word of the form $W E W$; and if $W E_{1} W, W E_{2} W \epsilon$ $B\left(T_{k}\right)$, then $W E_{1} W B W E_{2} W \in B\left(T_{k}\right)$, again by the intersection property. So $T_{k}$ is irreducible.

The zeta function of a transformation $S$ is

$$
\zeta(t)=\exp \left\{\sum_{n=1}^{\infty} \frac{N_{n}(S)}{n} t^{n}\right\},
$$

where $N_{n}(S)=\left|\left\{x: S^{n} x=x\right\}\right|$. For SFT's and sofic shifts, the zeta function is a computable invariant from which the periodic counts $N_{n}(S)$ (and thus also $\pi_{n}(S)$ ) can be recovered.

\section{The main result}

In the following lemma, we construct a bounded-to-one cover of $T$ by 'blowing up' an orbit of period $J$ into $k$ orbits whose periods are $J M_{1}, J M_{2}, \ldots, J M_{k}$.

(2.1) Covering Lemma. Suppose $T$ is an irreducible SFT of positive entropy with $\pi_{J}(T)>0$, and $M_{1}, \ldots, M_{k}$ are positive integers. Then there is an irreducible SFT $S$ such that the following conditions hold:

(a) there is a factor map $\phi: S \downarrow T$, and $h(S)=h(T)$;

(b) if $T$ is mixing, then $S$ is mixing;

(c) $\pi_{j}(S)=\pi_{j}(T) \quad$ whenever $j \neq J M_{i}$ for any $M_{i}$ and $j \neq J$;

(d) $\pi_{J}(S)=\pi_{J}(T)-J+J\left|\left\{i: M_{i}=1\right\}\right|$;

(e) if $N=J M_{i}$ for some $M_{i}>1$, then

$$
\pi_{N}(S)=\pi_{N}(T)+N\left|\left\{i: J M_{i}=N\right\}\right| .
$$

Proof. We must suffer some notation. Let $A=a_{1} \ldots a_{J}$ in $B_{J}(T)$ define an orbit with least period $J$ in $T$. Recoding if necessary, we may assume the $J$ symbols 
$a_{1}, \ldots, a_{J}$ are distinct, and $T$ is 1 -step. Given $x$ in $T$, let $x(\alpha \ldots \beta)$ be the (possibly infinite) substring of $x$ using $\left\{x_{i}: \alpha<i<\beta\right\}$; for example, $x(0 \ldots+\infty)=x_{1} x_{2} x_{3} \ldots$, and $x(1 \ldots 4)=x_{2} x_{3}$. Let $A^{\infty}$ denote the twosidedly infinite string formed by concatenating $A$. We say $x(\alpha \ldots \beta)$ is periodic- $A$ if it occurs in $A^{\infty}$; for example, if $A=123$, then the block 31 is periodic- $A$, and so is the left-infinite string ...312312312312. We say $x(\alpha \ldots \beta)$ is $A$-maximal if it is periodic- $A$, nontrivial (that is, $\beta>\alpha+1$ ), and $\{i: \gamma<i<\delta\} \supsetneqq\{i: \alpha<i<\beta\}$ implies $x(\gamma \ldots \delta$ ) is not periodic- $A$.

Now add to the alphabet of $T$ new symbols $a^{r}(t)$, for $1 \leq r \leq k$ and $1 \leq t \leq J M_{r}$. For each such $r$, define a block $A_{r}$ of length $J M_{r}$ by

$$
A_{r}=a^{r}(1) a^{r}(2) \ldots a^{r}\left(J M_{r}\right)
$$

Each of the blocks $A_{r}$ will define a new periodic orbit.

Two facts follow from the irreducibility and positive entropy of $T$. First, there is at least one symbol $a_{i}$ from $A$ which has a predecessor not equal to $a_{i-1}$ (if $i=1$, then by $a_{i-1}$ we mean $a_{J}$ ). Second, fixing a sufficiently large $L$, for each such $a_{i}$ we may partition the set of blocks of length $L$ which may precede $a_{i}$ but do not end in $a_{i-1}$ into $k$ non-empty sets $C_{r}\left(a_{i}\right), 1 \leq r \leq k$.

We now define $S$. A sequence $\bar{x}$ is in $S$ if and only if it can be formed from some $x$ in $T$ (this $x$ we call the 'parent' of $\bar{x}$ ) subject to the following three rules:

(1) $\bar{x}_{i}=x_{i} \quad$ if $x_{i} \not \subset\left\{a_{j}: 1 \leq j \leq J\right\}$;

(2) if $x(\alpha \ldots \beta)$ is $A$-maximal in $x$, with $\alpha<I<\beta$ and $x_{I}=a_{i}$, then

(i) $\bar{x}(\alpha \ldots \beta)$ is $A_{r}$-maximal in $\bar{x}$ for some $r$, and

(ii) $\bar{x}_{I}=a^{r}(t) \quad$ for some $t \equiv i \bmod J$;

(3) if $x(\alpha \ldots \beta)$ is $A$-maximal in $x$, with $\alpha$ finite and $x_{\alpha+1}=a_{i}$, then

(i) $\bar{x}_{\alpha+1}=a^{r}(i)$, where $r$ is determined by requiring

(ii) $x_{\alpha-L+1} \ldots x_{\alpha} \in C_{r}\left(a_{i}\right)$.

The required factor map $\phi$ is the natural 1-block map: $a^{r}(t) \mapsto a_{i}$ if $i \equiv t \bmod J$. The map $\phi$ takes $\bar{x}$ to its (unique) parent $x$. In forming $\bar{x}$ from $x$, all we have done is to replace stretches periodic in $A$ with stretches periodic in some $A_{r}$ Condition 2(ii) ensures that a new symbol can replace only one symbol from $T$, so that the map $(\bar{x} \rightarrow$ parent of $\bar{x})$ is a well-defined 1-block map. Conditions 2 and 3 ensure that only finitely many $\bar{x}$ can be formed from a given $x$, because they specify uniquely which $A_{r}$-maximal string may replace an $A$-maximal string which is not left-infinite in $x$. In particular, these conditions imply that $\phi$ is a bijection between the periodic points of $S$ and those of $T$, except for the $k+1$ orbits defined by $A$ and the $A_{r}$ Because for each $r$ there is some non-empty $C_{r}\left(a_{i}\right)$, all of the new orbits actually appear in $S$ (many variations of the construction will also guarantee this.) We leave to the reader the verifications that $\phi: S \downarrow T, S$ is an ( $L$-step) irreducible SFT, $h(S)=h(T)$ and $S$ is mixing if $T$ is mixing.

Remarks. Adler and Marcus showed in [1] that, given an MSFT $U$, there is an MSFT $T$ with a fixed point such that $h(T)=h(U)$. Given non-negative integers $a_{1}, \ldots, a_{n}$, one can apply the covering lemma finitely many times to this $T$ to produce an 
MSFT $S$ such that $h(S)=h(T)$ and $\pi_{i}(S)=i a_{i}$ if $1 \leq i \leq n$. That is, for any allowed entropy, any finite periodic pattern will occur in some MSFT of that entropy.

For the record, we note some properties of the factor map $\phi$ of (2.1) which we shall not need in this paper. The map $\phi$ is right closing ([4], [7]) and 1-1 a.e. If only one new orbit is added (that is, $k=1$ in the statement of the lemma), then $\phi$ is right-resolving ([1]).

We call a block $b_{1} \ldots b_{k} j$-periodic if $1 \leq i<i+j \leq k$ implies $x_{i}=x_{i+j}$.

(2.2) LeMma (Krieger). Let $S$ be a subshift. Given $k>N>1$, there is a closed open set $F$ such that

(1) the sets $S^{i} F, 0 \leq i<N$, are disjoint, and

(2) if $x \in S$ and $x_{-k} \ldots x_{k}$ is not a $j$-periodic block for any $j<N$, then

$$
x \in \bigcup_{-N<i<N} S^{i} F
$$

Proof. Given $k>N>1$, enumerate the allowed $(2 k+1)$-blocks which are not $j$ periodic for any $j<N$ as $C_{1}, \ldots, C_{L}$. Let $E_{i}=\left\{x: x_{-k} \ldots x_{k}=C_{i}\right\}$. Let $F_{1}=E_{1}$. Given $F_{j}$, let

Set $F=F_{L}$.

$$
F_{j+1}=F_{j} \cup\left(E_{j+1} \sim \bigcup_{-N<i<N} S^{i} F_{j}\right)
$$

The simplicity of Krieger's lemma is perhaps deceptive. The set $F$, whose definition typically requires many more than $2 k$ coordinates, incorporates a tremendous amount of combinatorial information.

(2.3) LEMMA. (1) Suppose $x$ and $y$ are twosidedly infinite sequences with periods $j$ and $k$, respectively, and for some $i$ the blocks $x_{i} \ldots x_{i+j+k-1}$ and $y_{i} \ldots y_{i+j+k-1}$ are equal. Then $x=y$.

(2) Suppose $x_{i} \ldots x_{l}$ is a block with $l-i \geq 2 N-3$. Then there is at most one sequence $\tilde{x}$ with period strictly less than $N$ such that

$$
\tilde{x}_{i} \ldots \tilde{x}_{l}=x_{i} \ldots x_{l} \text {. }
$$

Proof. (1) Using the assumed equality and the periodicities of $x$ and $y$, we find

$$
x_{i+j+k}=x_{i+k}=y_{i+k}=y_{i}=x_{i}=x_{i+j}=y_{i+j}=y_{i+j+k} \text {. }
$$

That is, $x_{i+j+k}=y_{i+j+k}$. An induction shows $x_{t}=y_{t}$ for all $t \geq i$. Similarly, $x_{t}=y_{t}$ for all $t \leq i$.

(2) Suppose there are two such $\tilde{x}$, and apply (1) for a contradiction.

Recall that $\phi: S \rightarrow T$ means that $\phi$ is a continuous, shift-commuting map from $S$ to $T$, while $\mathrm{s} \stackrel{\text { per }}{\longrightarrow} T$ means that the period of any periodic point of $S$ is divisible by the period of some periodic point of $T$.

(2.4) Extension Lemma. Suppose that $S, \bar{S}$ and $T$ are subshifts satisfying the following conditions:

(1) $T$ is a mixing subshift of finite type;

(2) $\bar{S}$ is a subshift of $S$; 
(3) there is a map $\bar{\phi}: \bar{S} \rightarrow T$;

(4) $S \stackrel{\text { per }}{\longrightarrow} T$.

Then $\bar{\phi}$ extends to a map $\phi: S \rightarrow T$.

Proof. Recoding if necessary, presume $\bar{\phi}$ is 1-block and $T$ is 1-step with transition length $N$. Use Krieger's lemma to find $k>2 N$ and a closed open set $F \subset S$ such that the following conditions hold:

(1) the sets $S^{i} F, 0 \leq i<N$, are disjoint;

(2) for any integer $i$, if $x \in S$ and $x \notin \bigcup_{-N<j<N} S^{j} F$, then $x_{i-k} \ldots x_{i+k}$ is a $j$-periodic block for some $j<N$;

(3) the number $k$ is large enough to ensure that, if $j$ is less than $N$ and a $j$-periodic string of length $2 k+1$ occurs in some $x$ in $S$, then that string defines a $j$-periodic orbit which actually occurs in $S$ (the existence of $k$ follows from compactness).

We pause to visualize (as in [6]) a segment of $x$ :

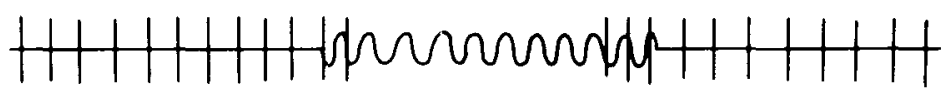

FigUre 1

The wavy part is $j$-periodic, $j<N$. The bars are markers placed at points of entry into $F$ : there is a bar at $x_{i}$ if $S^{i} x \in F$. Then (1) above tells us that in a sequence of symbols from $S$, there are at least $N-1$ unmarked symbols between successive markers. Similarly, (2) above tells us that if $x \in S$ and a string of length $2 N-1$ from $x$ has no markers, then that string is occurring in $x$ within a 'wavy' string of length $\geq 2 k+1$.

We will define the code $\phi$ in three parts.

I. Code between nearby markers. The 1-block map $\bar{\phi}$ induces for each $i$ a map $\bar{\phi}_{i}: B_{i}(\bar{S}) \rightarrow B_{i}(T)$. First, extend $\bar{\phi}_{1}: A(\bar{S}) \rightarrow A(T)$ arbitrarily to $\phi_{1}: A(S) \rightarrow A(T)$. Then, if $N<i \leq 2 N$, extend $\bar{\phi}_{i}$ to $\phi_{i}: B_{i}(S) \rightarrow B_{i}(T)$, requiring only that the first symbol of $\phi_{i}\left(b_{1} \ldots b_{i}\right)$ be $\phi_{1}\left(b_{1}\right)$ and that the last be $\phi_{1}\left(b_{i}\right)$. This is possible because $N$ is a transition length for $T$ and $i>N$. Now we can define $\phi$ between nearby markers. If $S^{i} x \in F$ and $S^{j} x \in F$, where $N \leq j-i \leq 2 N-1$, then

$$
(\phi x)_{i} \ldots(\phi x)_{j}=\phi_{j-i+1}\left(x_{i} \ldots x_{j}\right) .
$$

II. Periodic code. Since $S \stackrel{\text { per }}{\longrightarrow} T$, there is a shift-commuting map $p$ of points in $S$ of period less than $N$ to points in $T$ such that $p x=\bar{\phi} x$ if $x \in \bar{S}$. We will let $p$ determine $\phi$ inside periodic strings at locations at least $N$ symbols from a marker.

So, suppose $S^{i} x \notin \bigcup_{-N<j<N} S^{j} F$. By (3) above and lemma 2.3, there is a unique point $\tilde{x} \in S$ with period less than $N$ such that $\tilde{x}_{j}=x_{j}$ if $i-N<j<i+N$ : we define $(\phi x)_{i}=(p \tilde{x})_{i}$.

III. Transition code. Finally we define transitions between the periodic and aperiodic stretches. For each pair $(a, b)$ from $A(T) \times A(T)$ choose from $B_{N+1}(T)$ a block $\Phi(a, b)$ which begins with $a$ and ends with $b$. 
Suppose $S^{i} x \in F$, but $S^{i+j} x \notin F$ if $0<j<2 N$. Then we have already given the 'periodic' definition for $(\phi x)_{i+N}$. We define

$$
\begin{aligned}
(\phi x)_{i} \ldots(\phi x)_{i+N} & =\Phi\left(\phi_{1}\left(x_{i}\right),(\phi x)_{i+N}\right) \quad \text { if } x_{i} \ldots x_{i+N} \notin B_{N+1}(\bar{S}) \\
& =\bar{\phi}_{N+1}\left(x_{i} \ldots x_{i+N}\right) \quad \text { if } x_{i} \ldots x_{i+N} \in B_{N+1}(\bar{S}) .
\end{aligned}
$$

Similarly, suppose $S^{i} x \in F$, but $S^{i-j} \not \subset F$ if $0<j<2 N$. Then we define

$$
\begin{aligned}
(\phi x)_{i-N} \ldots(\phi x)_{i} & =\Phi\left((\phi x)_{i-N}, \phi_{1}\left(x_{i}\right)\right) \quad \text { if } x_{i-N} \ldots x_{i} \not B_{N+1}(\bar{S}) \\
& =\bar{\phi}_{N+1}\left(x_{i-N} \ldots x_{i}\right) \quad \text { if } x_{i-N} \ldots x_{i} \in B_{N+1}(\bar{S}) .
\end{aligned}
$$

The definition of $\phi$ is now complete. Since $k>N$, the above definitions overlap on at most a single coordinate, where they must agree. Since $T$ is a 1 -step SFT, $\phi$ is well defined: the assembly of blocks from the code for $\phi x$ must produce a point which is actually in $T$. The construction ensures that $\phi=\bar{\phi}$ on $\bar{S}$.

Remark. The method of the extension lemma is flexible. For example, if in the lemma $\bar{\phi}$ is injective, $h(S)<h(T)$ and for all $j, \pi_{j}(S) \leq \pi_{j}(T)$, then we can extend $\bar{\phi}$ to an embedding $\phi$ by coding markers into the image as Krieger did in [5]. Analogously, if in the lemma we add the assumptions that $\bar{S}$ is a proper subshift, $S$ is sofic and $h(S)>h(T)$, then we can extend $\bar{\phi}$ to a surjection $\phi: S \downarrow T$ with the help of Marcus' result.

(2.5) THEOREM (Main result). Suppose $S$ and T are irreducible subshifts of finite type, $S \stackrel{\text { per }}{\longrightarrow} T$ and $h(S)>h(T)$. Then $S \downarrow T$.

Proof. First suppose $S$ and $T$ are also mixing. Since $h(S)>h(T)$, it follows that

$$
\lim \left(\pi_{j}(S)-\pi_{j}(T)\right)=+\infty \text {. }
$$

So, we may use the covering lemma finitely many times to find an SFT $\bar{S}$ such that $\bar{S} \downarrow T, h(\bar{S})=h(T)$ and for all $j, \pi_{j}(\bar{S}) \leq \pi_{j}(S)$. By Krieger's Embedding Theorem, we may assume $\bar{S}$ is a subshift of $S$. Apply the extension lemma to extend $\bar{S} \downarrow T$ to $S \downarrow T$.

If $S$ and $T$ need not be mixing, let $k$ and $l$ be the ergodic periods of $S$ and $T$. Pick a prime $p$ greater than $l$ such that $S$ has a point of period $p k$. Since $S \stackrel{\text { per }}{\longrightarrow} T$, some multiple of $l$ must divide $p k$, hence $l$ divides $k$.

Let $E$ and $F$ be cyclically moving subsets for $S$ and $T$. Since $l$ divides $k$, the restriction of $T^{k}$ to $F$ is well-defined and mixing. So by the first paragraph of the proof, we can find $\phi:\left(\left.S^{k}\right|_{E}\right) \downarrow\left(\left.T^{k}\right|_{F}\right)$. Then there is a unique extension of $\phi$ to the full domain of $S$ which factors $S$ onto $T$.

Theorem 2.5 fails if the requirement of irreducibility is relaxed for either $S$ or $T$.

(2.6) Corollary (Lifting MSFT's). Suppose $S$ and $T$ are subshifts satisfying the following conditions:

(1) $T$ is a mixing SFT;

(2) $S$ contains an SFT $\tilde{S}$ such that $h(\tilde{S})>h(T)$, (for example, $S$ is sofic and $h(S)>h(T))$;

(3) $S \stackrel{\text { per }}{\longrightarrow} T$.

Then $S \downarrow T$. 
Proof. By restriction to an irreducible SFT of equal entropy, we may assume $\tilde{S}$ is irreducible. Since $S \stackrel{\text { per }}{\longrightarrow} T$, we know $\tilde{S} \stackrel{\text { per }}{\longrightarrow} T$; so $\tilde{S} \downarrow T$. Use the extension lemma to extend to $S \downarrow T$.

In (2.6), $S$ need not even be transitive. However, example 3.5 shows that the mixing constraint on $T$ cannot be relaxed.

(2.7) Corollary. Suppose $S$ and $U$ are irreducible SFT's with equal and positive entropy. Then the following conditions are equivalent.

(1) Every factor of $U$ of lower entropy is also a factor of $S$.

(2) Whenever $\pi_{J}(S)>0$, there is some $j$ dividing $J$ such that $\pi_{j}(U)>0$.

Proof. (1) $\Rightarrow(2)$. Suppose $\pi_{J}(S)>0$ and there is no $j$ dividing $J$ for which $\pi_{j}(U)>0$. Pick some SFT $T$ such that $U \downarrow T$ and $h(T)<h(U)$. Now apply the covering lemma to find an SFT $\bar{T}$ such that $\bar{T} \downarrow T, h(\bar{T})=h(T), U \stackrel{\text { per }}{\longrightarrow} \bar{T}$ but $\pi_{j}(\bar{T})=0$ if $j$ divides $J$. Then $U \downarrow \bar{T}$ but $S$ cannot factor onto $\bar{T}$.

(2) $\Rightarrow(1)$. Suppose $U \downarrow T$ where $h(T)<h(U)$. We must show $S \downarrow T$; this is not immediate from (2.5), because $T$ might be sofic. It suffices to intercept the factor map $U \downarrow T$ to produce an SFT $\bar{T}$ such that $h(\bar{T})<h(U)$ and $U \downarrow \bar{T} \downarrow T$ : then by (2.5), $S \downarrow \bar{T}$. We leave this as an exercise.

For help in the classification of MSFT's, one might look to the classes of lowerentropy factors or subshifts for invariants. Krieger's Embedding Theorem show: that the class of subshifts can tell us no more than we already know from the zeta function. Corollary 2.7 shows the same for the lower-entropy factors.

In the next section, we find the sofic situation is more complicated.

\section{The sofic situation}

We begin by considering examples which limit the extension of previous results to sofic shifts. The following example is due essentially to Brian Marcus; we have modified his example to control the zeta function.

(3.1) Example. We exhibit a mixing sofic shift with finite-type zeta function lacking periodically allowed finite-type subshifts and extensions.

Let $T$ be the strictly sofic shift on alphabet $\left\{a_{1}, a_{2}, b\right\}$ in which the following blocks are disallowed:

(1) $a_{i} a_{j}, \quad$ for $1 \leq i, j \leq 2$;

(2) $a_{i} b^{n} a_{j}, \quad$ for $n \neq i \bmod 2,1 \leq i, j \leq 2$.

Let $U$ be the SFT on alphabet $\{a, b\}$ in which the block $a a$ is disallowed. Then $T \downarrow U$ by the 1-block map $a_{i} \mapsto a, b \mapsto b$. This factor map is a bijection on the periodic points, so $T$ and $U$ have the same zeta function.

Now suppose $\phi: S \rightarrow T$, where $S$ is an irreducible SFT with a fixed point ( $x$, say, with $x_{n}=1$ for all $n$ ): we claim $\phi$ must map all of $S$ onto the fixed point of $T$. Let $y$ be the fixed point of $T$; clearly $\phi x=y$. If the image contains another point, then the irreducibility of $S$ implies the existence of blocks $B, C, D, E$ and positive integers $j, k$ for which the following hold:

(1) $\phi$ maps the orbit $1^{\infty} B 1^{k} C 1^{\infty}$ to the orbit $b^{\infty} D b^{j} E b^{\infty}$; 
(2) $\phi$ maps $1^{\infty} B 1^{k+1} C 1^{\infty}$ to $b^{\infty} D b^{j+1} E b^{\infty}$;

(3) some $a_{i}$ occurs in $D$ and in $E$.

This is a contradiction, because $D b^{j} E$ and $D b^{j+1} E$ cannot both occur in $T$.

(3.2) Example. We show that two mixing sofic shifts with the same zeta function may have different lower-entropy factors.

We describe sofic $S$ and $\tilde{S}$ by the following diagrams:



$S$

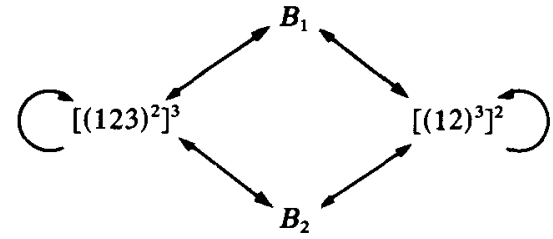

$\xi$

Figure 2

The diagrams indicate what blocks may follow other blocks. There $B_{1}$ and $B_{2}$ are distinct blocks on new letters, of length 5, say.

The zeta functions of $S$ and $\tilde{S}$ are the same. Basically, we have two distinct six-blocks, 121212 and 123123; one may occur between $B_{i}$ 's $2 n$ times, the other $3 n$ times, $n \geq 1$. All periodic orbits of $S$ and $\tilde{S}$ except $(12)^{\infty}$ and $(123)^{\infty}$ occur by such sandwiching, and can be put in a natural 1-1 correspondence.

Now obtain $T, h(T)<h(S)$, from $S$ by identifying $B_{1}$ and $B_{2}$ as $B$. Suppose $\tilde{S} \downarrow T$. Clearly $(12)^{\infty} \rightarrow(12)^{\infty}$. For a large $k$, then, an orbit $(12)^{\infty} B_{1}(12)^{k} B_{1}(12)^{\infty}$ must go to some orbit $(12)^{\infty} C(12)^{j} C(12)^{\infty}$, where $B$ occurs in $C$ and $j>0$, and also $(12)^{\infty} B_{1}(12)^{k+6} B_{1}(12)^{\infty}$ must go to $(12)^{\infty} C(12)^{j+6} C(12)^{\infty}$.

But this is a contradiction, since $j$ can increase only by increments of 9 .

In the examples, factor and embedding maps into mixing sofic shifts were ruled out by contradictory congruence conditions on allowed periodic block counts. In fact, nothing else can go wrong. To make this claim precise, we define a class of periodic points (receptive points) on which such congruence pathology is ruled out. Suppose a point $x$ has least period $J$ for a mixing sofic shift $S$, and set $A=x_{0} \ldots x_{J-1}$. We say $x$ is receptive if there exist magic words $W_{1}$ and $W_{2}$ such that the blocks $W_{1} A^{n} W_{2}$ are allowed for $n \geq 1$. (There is an equivalent condition, less useful for coding: given $S$-blocks $E$ and $F$, there exist blocks $\bar{E}$ and $\bar{F}$ such that $E \bar{E} A^{n} \bar{F} F$ is an $S$-block if $n \geq 1$.) It is not enough to assume that there exist blocks $B$ and $C$ not periodic- $A$ such that $B A^{n} C$ is allowed for $n \geq 1$. For example, there might still exist a block $D$ such that $B A^{n} C D$ is allowed only for odd $n$. Difficulties of this sort are ruled out by the intersection property of magic words.

(3.3) ThEOREM (Lifting Mixing Sofic Shifts). Suppose S and T are subshifts satisfying the following conditions:

(1) $T$ is mixing sofic; 
(2) $S$ contains an SFT $\tilde{S}$ such that $h(\tilde{S})>h(T)$, (for example, $S$ is sofic and $h(S)>h(T))$

(3) if $\pi_{j}(S)>0$, then $T$ contains a receptive point whose period divides $j$. Then $S \downarrow T$.

Proof. As in corollary 2.6, we will find a subshift $\bar{S}$ of $S$ and a map $\bar{\phi}: \bar{S} \downarrow T$, which we will extend to $\phi: S \downarrow T$. Because $T$ need not be finite type, we must work harder to ensure the blocks obtained in $T$ by linking up blocks from the code are actually allowed for $T$.

First we find $\bar{\phi}$. Pick an MSFT $U$ such that $U \downarrow T$ and $h(U)=h(T)$. Find an irreducible SFT $\bar{S}$ in $\tilde{S}$ such that $\bar{S} \stackrel{\text { per }}{\longrightarrow} U$ and $h(\bar{S})>h(U)$. Then $\bar{S} \downarrow U \downarrow T$ gives $\bar{\phi}: \bar{S} \downarrow T$. Recoding, we may assume $\bar{S}$ is 1 -step and $\bar{\phi}$ is 1 -block. The proof of theorem 2.5 shows that we may assume the map $\bar{S} \downarrow U$ maps each cyclically moving subset of $S$ onto $U$. Consequently, since $U$ is mixing, there is a positive integer $N(\bar{S})$ for which the following condition holds. Suppose $a \in A(\bar{S}), B \in B(T)$ and $l \geq N(\bar{S})$; then there are blocks $B_{1}, B_{2}, C_{1}$ and $C_{2}$ in $B(\bar{S})$ such that

(i) $\bar{\phi}\left(B_{1}\right)=\bar{\phi}\left(B_{2}\right)=B$;

(ii) $a C_{1} B_{1}$ and $B_{2} C_{2} a$ are in $B(\bar{S})$; and

(iii) $C_{1}$ and $C_{2}$ have length $l$.

Let $W$ be a magic word in $T$. Let $N(T)$ be a transition length for $T$. Let $N=\max \{N(\bar{S}), N(T)+|W|\}$. Choose $k$ large enough so that we may define a marking set $F=F(N, k)$ satisfying the conditions (1), (2) and (3) stated in the proof of the extension lemma (2.4). For later convenience, we also require $k>6 N$.

To see how the pieces of the code $\phi$ will fit together, use the following picture of a sample segment of some sequence $x$ from $S$.

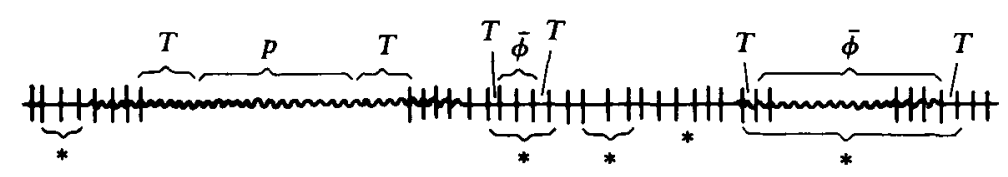

FIGURE 3

The vertical bars mark the coordinates $x_{i}$ such that $S^{i} x \in F$. The wavy stretches represent blocks of length greater than $2 k$ which are $j$-periodic for some $j<N$. We call $x_{i} \ldots x_{j}$ a marker block in $x \cdot$ if $S^{i} x \in F, S^{j} x \in F$ and $S^{t} x \notin F$ for $i<t<j$. In the picture, an asterisk appears below marker blocks from $B(\bar{S})$. We will use several rules to define the code; symbols above marker blocks indicate where the different rules are used. The marker blocks without symbols overhead are coded by rule $W$.

There will be three fundamental rules: $\bar{\phi}, p$ and $W$. Rule $\bar{\phi}$ is used on long marker-free stretches of symbols from $A(\bar{S})$, and on marker blocks in $B(\bar{S})$ flanked on each side by a marker block or wavy stretch from $\bar{S}$; this rule agrees with $\bar{\phi}$ and guarantees $\phi$ is an extension of $\bar{\phi}$. Rule $W$ is used on all sufficiently short marker blocks for which rule $\bar{\phi}$ (or a transition rule) is not used. Rule $p$ is used inside long marker-free stretches which do not occur in $\bar{S}$; this rule ensures points of small period in $S \sim \bar{S}$ are mapped to receptive points. 
A transition code is used on a marker block from $B(\bar{S})$ which is flanked by blocks to which rules $\bar{\phi}$ and $W$ apply. Another transition code is used on initial and terminal segments of long marker-free stretches within which rule $p$ is used. We guarantee that the blocks we get in $T$ by gluing together the fundamental-rule blocks with the transition blocks are well-defined by coding appropriate magic words into the transition blocks.

Notice that if $x_{0} \ldots x_{2 k}$ is $j$-periodic for some $j<N$, but $x_{-1} \ldots x_{2 k}$ is not, then $S^{i} x \in F$ for at least three $i$ such that $0<i<6 N$; this is because $k>6 N$. That is, we see at least two marker blocks inside a 'wavy' stretch by the left boundary of the stretch; the same is true on the right. This convenience ensures that we never have to code a transition between rule $p$ and rule $\bar{\phi}$. Similarly, the statement of the transition rule between rule $W$ and rule $\bar{\phi}$ is simplified, because this rule will apply only to marker blocks flanked by marker blocks of length less than $2 N$.

We now define the code $\phi$.

I. Rule $\bar{\phi}$. Recall $\bar{\phi}$ is 1-block. We define $(\phi x)_{i}=\bar{\phi}\left(x_{i}\right)$ if one of the following conditions hold.

Condition 1. There exists an integer $I$ such that the following hold:

(i) $I<i<I+2 N$;

(ii) $x_{I} \ldots x_{I+2 N} \in B(\bar{S})$;

(iii) $S^{t} x \notin F$ if $I<t<I+2 N$.

Condition 2. There are integers $a<b<c<d$ such that the following hold:

(i) $b \leq i \leq c$;

(ii) $x_{a} \ldots x_{d} \in B(\bar{S})$;

(iii) $S^{b} x \in F$ and $S^{c} x \in F$;

(iv) $S^{a} x \in F$ or $a+2 N \leq b$;

(v) $S^{d} x \in F$ or $c+2 N \leq d$.

II. Rule $p$. Let $p$ be a shift-commuting map from the points $\mathscr{P}$ in $S \sim \bar{S}$ of period less than $N$ to receptive points in $T$. Given $R>N$, let $M=2 R+N$. Suppose there are integers $i, I$ and $J$ for which the following conditions hold:

(i) $J-I \geq M$;

(ii) $I+R<i<J-R$;

(iii) $S^{t} x \notin F$ if $I<t<J$;

(iv) $x_{i} \ldots x_{i+N} \not \subset B(\bar{S})$.

Then we define $(\phi x)_{i}=(p \tilde{x})_{i}$, where $\tilde{x}$ is the unique point in $\mathscr{P}$ such that $\tilde{x}_{I} \ldots \tilde{x}_{J}=$ $x_{I} \ldots x_{j}$. (Because $k$ satisfies condition (3) from the extension lemma, $\tilde{x}$ exists; by lemma $2.3, \tilde{x}$ is unique.)

This rule determines the code inside long periodic strings of $S \sim \bar{S}$ at locations more than $R$ symbols from a marker. The number $R$, determined below, is large enough to ensure room for a transitional code between rule $p$ and rule $W$.

III. Rule $W$. For each $l \geq N$, pick a block $C_{l}$ of length $l-|W|$ such that $W C_{l} W \in$ $B(T)$; this is possible because $N \geq N(T)+|W|$. Then we define $(\phi x)_{i} \ldots(\phi x)_{j-1}=$ $W C_{j-i}$ if $x_{i} \ldots x_{j}$ is a marker block in $x$ satisfying the following conditions:

(i) $j-i<M$; 
(ii) rule $\bar{\phi}$ is not used to define $(\phi x)_{i} \ldots(\phi x)_{j}$;

(iii) if $i-2 N<h<i$ and $x_{h} \ldots x_{i}$ is a marker block in $x$, then rule $\bar{\phi}$ is not used to define $(\phi x)_{h} \ldots(\phi x)_{i}$;

(iv) if $j<l<j+2 N$ and $x_{j} \ldots x_{l}$ is a marker block in $x$, then rule $\bar{\phi}$ is not used to define $(\phi x)_{j} \ldots(\phi x)_{l}$.

The intersection property of magic words guarantees that blocks assembled in $T$ by concatenating pieces of the code from rule $W$ must actually occur in $T$.

IV. Transition between rule $W$ and rule $\bar{\phi}$. Because $N \geq N(\bar{S})$, for each $a \in A(\bar{S})$ and $j \geq N$ we may choose a word $E(j, a)$ of length $j$ from $B(\bar{S})$ such that $E(j, a) a \in$ $B_{j+1}(\bar{S})$ and $\bar{\phi}(E(j, a) a)$ begins with $W$. Define

$$
(\phi x)_{i} \ldots(\phi x)_{j-1}=\bar{\phi}\left(E\left(j-i, x_{j}\right)\right)
$$

if there exist integers $h$ and $l$ such that the following hold:

(i) $h<i<j<l$, with $l-h<6 N$;

(ii) $x_{h} \ldots x_{i}, x_{i} \ldots x_{j}$ and $x_{j} \ldots x_{l}$ are marker blocks in $x$,

(iii) $x_{h} \ldots x_{i} \not B(\bar{S})$;

(iv) $x_{i} \ldots x_{l} \in B(\bar{S})$;

(v) Rule $\bar{\phi}$ is used to determine $(\phi x)_{j} \ldots(\phi x)_{l}$.

This defines transitions from rule $W$ to rule $\bar{\phi}$. The transition from rule $\bar{\phi}$ to rule $W$ is similar.

V. Transition between rule $p$ and rule $W$. Pick one representative $y$ from each of the receptive orbits in the image of $p$, and let $A=y_{0} \ldots y_{j-1}$, where $j$ is the least period of $y$. By hypothesis, there exist magic words $W_{1}$ and $W_{2}$, depending on $y$, such that $W_{1} A^{n} W_{2}$ is allowed for all positive $n$. We may assume that $W_{1}$ and $W_{2}$ have the same length $L$ for each of these $y$. Also, for each $l$ between $|W|+L+N(T)$ and $|W|+L+N(T)+N$, we may choose blocks $D_{l}$ and $E_{l}$ such that $W D_{l} W_{1} \in B_{l}(T)$ and $W_{2} E_{l} W \in B_{l}(T)$. We will code the following picture:

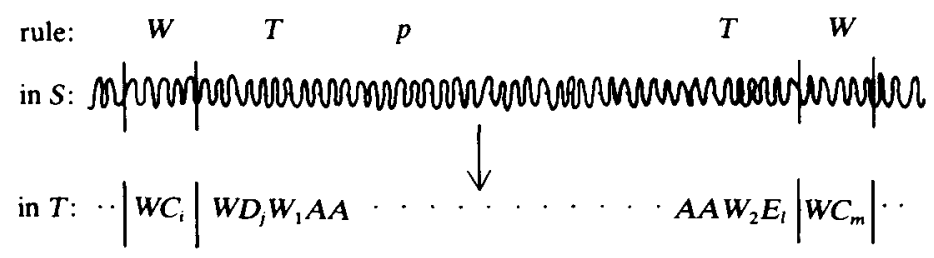

FIGURE 4

Then the intersection property of the magic words will guarantee that rules $W$ and $p$ are hooked up in a well defined way. The point of the variable length $C$ 's and $D$ 's is to let us finish the transition in synchrony with $A$.

So, suppose $S^{i} x \in F$, but $S^{t} x \notin F$ for $i<t<i+M$, and $x_{i} \ldots x_{i+N} \notin B(\bar{S})$. Let $\tilde{x}$ be the point in $\mathscr{P}$ such that $\tilde{x}_{i} \ldots \tilde{x}_{i+M}=x_{i} \ldots x_{i+M}$. From the orbit of $p \tilde{x}$, pick the distinguished $y$ and $A=y_{0} \ldots y_{j-1}$ chosen above. Pick the least $t$ such that

(i) $|W|+L+N(t) \leq t \leq|W|+L+N(T)+N$, and

(ii) $(p \tilde{x})_{t} \ldots(p \tilde{x})_{t+j-1}=A$. 
Then define

$$
(\phi x)_{i} \ldots(\phi x)_{i+t-1}=W C_{t} W_{1}
$$

and

$$
(\phi x)_{t} \ldots(\phi x)_{i+R}=(p \tilde{x})_{t} \ldots(p \tilde{x})_{i+R} .
$$

This codes the transition from rule $W$ to rule $p$. The transition from rule $p$ to rule $W$ is similar. Let $R=l(W)+2 N+L$, and we have room for the transitions. Since $M=2 R+N$, the definitions are consistent, and at least one copy of $A$ must appear between $W_{1}$ and $W_{2}$ in the image of an application of the transition rule $V$.

The previous theorem lets us characterize those mixing sofic shifts which behave like MSFT's with respect to higher-entropy sofic extensions. We say a sofic shift $T$ is receptive if whenever $S$ is sofic with $h(S)>h(T)$ and $S \stackrel{\text { per }}{\longrightarrow} T$, then $S \downarrow T$. For example, the even system of Weiss $([8])$ is the sofic shift obtained from the 2-shift (on symbols 1 and 2, say) by disallowing the blocks $2 l^{k} 2$ for which $k$ is odd. This shift is receptive because it contains a receptive fixed point.

(3.4) Corollary. Suppose Tis a mixing sofic shift. Then the following conditions are equivalent.

(1) $T$ is receptive.

(2) If $\pi_{j}(T)>0$, then $T$ has a receptive point with period dividing $j$.

Proof. $(2) \Rightarrow(1)$. Apply theorem 3.3.

$(1) \Rightarrow(2)$. Suppose $\pi_{J}(T)>0$ and if $k<J$ divides $J$ then $\pi_{k}(T)=0$. We must show $T$ has a receptive point of period $J$. Construct an MSFT $\bar{T}$ such that $h(\bar{T})>$ $h(T), \bar{T} \stackrel{\text { per }}{\longrightarrow} T$ and $\pi_{J}(\bar{T})>0$. (For example, obtain $\bar{T}$ from any MSFT with a fixed point and entropy greater than $h(T)$ by repeated use of the covering lemma.)

So, $\bar{T} \downarrow T$. We may assume the factor map $\phi$ is one-block and $\bar{T}$ is one-step. If $\bar{C}$ is a block for $\bar{T}$, we denote $\phi \bar{C}$ by $C$. Find $J$-blocks $\bar{A}, A$ defining orbits of period $J$. Find $\bar{W}$ so that $W$ is a magic word for $T$. Find blocks $\bar{E}, \bar{F}$ so that $\bar{W} \bar{E} \bar{A} \bar{F} \bar{W}$ is allowed for $\bar{T}$. Hence $\bar{W} \bar{E} \bar{A}^{n} \bar{F} \bar{W}$ is allowed for $\bar{T}$ and so $W E A^{n} F W$ is allowed for $T$ for all $n \geq 1$. But $W E$ and $F W$ are magic words.

Corollary 2.7 extends to mixing receptive sofic shifts; in this case, to prove $S \downarrow T$ given $U \downarrow T$, adjust the proof of theorem 3.3. (The new wrinkle is that, where receptive points in $T$ were used in the proof of (3.3), we now use points in $T$ which are images of appropriate receptive points in $U$.) In particular, two mixing receptive sofic shifts with the same zeta function have the same factors of lower entropy.

The following example shows one way in which (2.4), (2.6), (3.3) and (3.6) may fail if the range shift $T$ is not assumed to be mixing.

(3.5) Example. We will exhibit a transitive sofic shift $S$ such that there can be no continuous shift-commuting map $\phi$ from $S$ into any irreducible SFT $T$ with ergodic period two. With the covering lemma, it is easy to construct such a $T$ for which

(i) $S \stackrel{\text { per }}{\longrightarrow} T$ and $h(S)>h(T)$, or

(ii) for all $j, \pi_{j}(S) \leq \pi_{j}(T)$ and $h(S)<h(T)$. 
Thus conditions on periodic points and entropy fail to ensure the existence of various maps into non-mixing SFT's.

Let the graph in figure 5 give the allowed transitions for an irreducible period-two SFT $U$ on the five symbols $1^{a}, 3,2^{a}, 1^{b}$ and $2^{b}$.

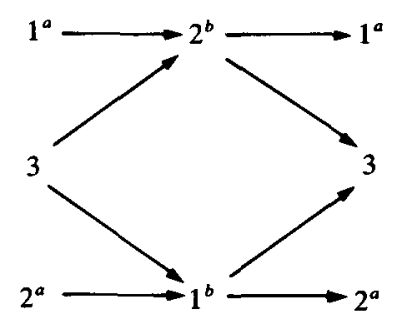

FIGURE 5

Some symbols are repeated in the graph for clarity. Define $S$ as the factor obtained by the following 1 -block map: $1^{a}, 1^{b} \mapsto 1 ; 2^{a}, 2^{b} \mapsto 2 ; 3 \mapsto 3$. This map identifies the orbits $\left(1^{a} 2^{b}\right)^{\infty}$ and $\left(1^{b} 2^{a}\right)^{\infty}$ but collapses no other points. In $S$, we see blocks like $3(12)^{n} 13$ and $3(21)^{n} 23$, and every periodic point has even period.

Suppose $T$ is an irreducible, period-two SFT and $\phi: S \rightarrow T$. By taking the inverse image of the cyclically moving subsets of $T$, we find that $S$ must be a disjoint union of clopen, $S^{2}$-invariant sets $X_{1}$ and $X_{2}$ such that $S\left(X_{1}\right)=X_{2}$ and $S\left(X_{2}\right)=X_{1}$. We may suppose the point $y$ of the orbit $(12)^{\infty}$ with 1 's on even coordinates is in $X_{1}$, and $S y \in X_{2}$. Since $y$ cannot be an accumulation point of $X_{2}$, and $S y$ cannot be an accumulation point of $X_{1}$, there must be some positive integer $N$ for which the following hold:

(1) if $n \geq N, x \in X_{1}$ and $x_{i} \ldots x_{i+2 n-1}=(12)^{n}$, then $i$ is even;

(2) if $n \geq N, x \in X_{2}$ and $x_{i} \ldots x_{i+2 n-1}=(12)^{n}$, then $i$ is odd.

This contradicts $S=X_{1} \cup X_{2}$. For example, no point of the orbit $\left(3(12)^{N} 13(21)^{N} 2\right)^{\infty}$ can satisfy (1) or (2).

(3.6) THEOREM (Embeddings into Sofic Shifts). Suppose S and Tare subshifts which satisfy the following conditions:

(1) $S$ is mixing sofic;

(2) for every $j, S$ has at least $\pi_{j}(T)$ receptive points of least period $j$;

(3) $h(S)>h(T)$.

Then $T$ is isomorphic to a subshift of $S$.

Proof. Since $S$ is mixing and $h(S)>h(T), S$ contains an MSFT $U$ such that $h(U)>$ $h(T)$ and $U$ contains a magic word. We could apply Krieger's Theorem to embed $T$ in $U$ and be done, except that $U$ might be short a finite number of periodic points. So it suffices to show, given an MSFT $U$ in $S$ containing a magic word $W$, and a receptive point $y$ not in $U$, that there is an MSFT $\bar{U}$ in $S$ which contains $U$ and $y$.

Let $A=y_{0} \ldots y_{J-1}$, where $J$ is the (least) period of $y$. Because $y$ is receptive, there are magic words $W_{1}, W_{2}$ such that $W_{1} A^{n} W_{2} \in B(S)$ for $n \geq 1$. Let $U$ be 
$N$-step. Pick blocks $C, D$ such that $W C W_{1}, W_{2} D W \in B(S)$. Pick

$$
M>\max \left\{J, N,\left|W C W_{1}\right|,\left|W_{2} D W\right|\right\} .
$$

Pick $B, E \in B_{5 M}(U)$ such that $B W, W E \in B(U)$. Now define a $(5 M-1)$-step SFT $\bar{U}$ by letting $B_{5 M}(\bar{U})$ be the union of $B_{5 M}(U)$ and all $5 M$-blocks which occur in the word

$$
Q=B W C W_{1} A^{5 M} W_{2} D W E
$$

Then $\bar{U}$ is an MSFT containing $y$ and $U$. We must check that $\bar{U}$ is a subshift of $S$. Let $Q=q_{1} \ldots q_{k}$. Suppose $x \in \bar{U}, F \in B_{5 M}(\bar{U}) \sim B_{5 M}(U)$ and $F$ is not periodic- $A$. Then there is a unique $I$ such that $q_{I} \ldots q_{I+5 M-1}=F$. Also, if $x_{t} \ldots x_{t+5 M-1}=F$, then $x_{t-1}=q_{I-1}$ and $x_{t+5 M}=q_{I+5 M}$. Therefore, if $F$ occurs in $x$, then $F$ occurs in $x$ within one of two blocks:

(1) a block $G W C W_{1} A^{\prime}$, where $G \in B(U), A^{\prime}$ is periodic- $A$ and $\left|A^{\prime}\right|,|G| \geq 3 M$ (this block is the smallest sub-block $q_{i} \ldots q_{j}$ of $B W C W_{1} A^{5 M}$ such that $j-i \geq 5 M$, $q_{i} \ldots q_{i+5 M-1} \in B_{5 M}(U)$ and $q_{j-5 M+1} \ldots q_{j}$ is periodic- $\left.A\right)$;

(2) a block $A^{\prime \prime} W_{2} D W H$, where $H \in B(U), A^{\prime \prime}$ is periodic- $A$ and $\left|A^{\prime \prime}\right|,|H| \geq 3 M$ (obtained similarly from the right side of $Q$ ).

Consequently, if two $A$-maximal stretches of length $\geq M$ occur in $x \in \bar{U}$, with no such intervening stretches, then the intervening stretch occurs in $x$ within a word $A^{\prime \prime} W_{2} D W X W C W_{1} A^{\prime}$, for some $U$-word $X$ of length greater than $M$ (hence greater than $N$ ). From the intersection property of magic words and the fact that $U$ is an $N$-step SFT contained in $S$, we see that such a word must be in $B(S)$. Similarly, all $\bar{U}$-words are $S$-words, and $\bar{U}$ is a subshift of $S$.

Theorem 3.6 lets us characterize those mixing sofic shifts which behave like MSFT's with respect to embeddings. We say that a subshift $S$ is inclusive if it satisfies Krieger's Embedding Theorem: that is, if $T$ is a subshift with $h(T)<h(S)$ and for all $j, \pi_{j}(T) \leq \pi_{j}(S)$, then $T$ is isomorphic to a subshift of $S$. For example, the even system of Weiss is not inclusive: (exactly) one of its periodic points is not receptive. If $S$ is mixing sofic with no congruence constraints on the iteration of periodic blocks, then $S$ must be inclusive; but this condition is not necessary for $S$ to be inclusive. For example, let $S$ be the subshift of the 3 -shift defined by excluding all blocks $21^{\mathrm{n}} 2$ for which $n$ is odd.

(3.7) Corollary. Suppose $S$ is a mixing sofic shift. Then the following conditions are equivalent.

(1) $S$ is inclusive.

(2) Every periodic point of $S$ is receptive.

Proof. (2) $\Rightarrow$ (1). Apply (3.6).

$(1) \Rightarrow(2)$. Suppose $x$ is periodic for $S$. We must show that $x$ is receptive. It suffices to show that $x$ is contained in some MSFT contained in $S$ for which there is some allowed block $W$ which is a magic word for $S$. Trivially, we may suppose $h(S)>$ 0 -otherwise $S$ is just a fixed point.

Let $J$ be the minimal period of $x$, and let $y$ be a point of minimal period $K$ such that $y_{1} \ldots y_{K}$ is a magic word for $S$. Construct $T$ an MSFT such that 
$h(T)<h(S), \pi_{J}(T)=\pi_{J}(S), \pi_{K}(T)=\pi_{K}(S)$ and for all $j, \pi_{j}(T) \leq \pi_{j}(S)$. (For example, obtain $T$ from any MSFT with a fixed point and entropy less than $h(S)$ by repeated use of the covering lemma.) Since $S$ is inclusive, $T$ is isomorphic to a subshift of $S$; but the embedding of $T$ into $S$ must contain $x$ and $y$.

The theorems (3.3) and (3.6) provide sufficient but not necessary conditions. Can the existence of a factor map/embedding between mixing sofic shifts of unequal entropy be characterized in terms of some tractable conditions on the periodic points?

I am grateful to Bruce Kitchens and Brian Marcus for encouragement and instruction. Especially I thank Doug Lind, who has guided me in ergodic theory, and suggested numerous improvements to an earlier version of this paper.

\section{REFERENCES}

[1] R. L. Adler \& B. Marcus. Topological Entropy and Equivalence of Dynamical Systems. Memoirs Amer. Math. Soc. 219 (1979).

[2] M. Denker, C. Grillenberger \& K. Sigmund. Ergodic Theory on Compact Spaces. Lecture Notes in Math. 527. Springer-Verlag: Berlin, 1976.

[3] R. Fischer. Graphs and Symbolic Dynamics. Topics in Information Theory (Second Colloq., Keszthely, 1975), (ed. I. Csiszar \& P. Elias), pp. 229-244. Colloq. Math. Soc., Janos Bolyai, Vol. 16, NorthHolland, Amsterdam, 1977.

[4] B. P. Kitchens. Ph.D. Thesis, University of North Carolina, Chapel Hill (1981).

[5] W. Krieger. On the subsystems of topological markov chains. Ergod. Th. \& Dynam. Sys. 2, (1982), 195-202.

[6] W. Krieger. On the Subsystems of Topological Markov Chains. Talk given at AMS conference 'Ergodic Theory and Applications', Durham, New Hampshire, U.S.A., June 1982. Unpublished.

[7] B. Marcus. Sofic systems and encoding data. Preprint, (Department of Mathematics, University of North Carolina, Chapel Hill (1982)).

[8] B. Weiss. Subshifts of finite type and sofic systems. Monatsh. Math. 77, (1973), 462-474. 